\title{
Computation of psychophysical thresholds using the probit technique
}

\author{
HARRIS R. LIEBERMAN \\ Massachusetts Institute of Technology, Cambridge, Massachusetts
}

\begin{abstract}
Probit analysis is a statistical technique that can be used for the computation of psychophysical thresholds, although it was originally developed for the analysis of pharmacological and toxicological data. Probit analysis may be applied to psychophysical data if the method of constant stimuli has been used for data collection. A computer program written in APPLESOFT BASIC that performs probit analyses on such psychophysical data is presented and explained.
\end{abstract}

Probit analysis is a statistical technique for the analysis of data from insecticidal, pharmacological, and toxicological assays (Bliss, $1935^{1}$; Garwood, 1941; Finney, 1962). It is usually applicable when the dependent variable in such assays is binomially distributed (quantal). Probit analysis has been adapted by psychologists for use in the analysis of binomially distributed psychophysical data (Bock \& Jones, 1968; Sakitt, 1974; Yang, Clark, Ngai, \& Berkowitz, 1979). When a bioassay is being performed to assess the potency of an experimental agent, such as an insecticide or toxin, the substance is administered to groups of experimental organisms in a range of dosages. The proportion of organisms responding to the substance at each of the dosage levels is the dependent variable of the experiment. The determination of whether a given organism responds to this substance must be quantal, that is, all or nothing.

The quantity of interest in a bioassay usually is the $\mathbf{L D}_{\mathbf{5 0}}$ or $\mathrm{ED}_{\mathbf{5 0}}$, the dose of the substance that will affect $50 \%$ of the organisms tested. Probit analysis is a technique for computing $\mathrm{LD}_{50}$ or $\mathrm{ED}_{50}$, as well as certain related parameters.

The statistical methodology developed by Finney (1962) for the analysis of this type of toxicological experiments is also applicable to psychophysical experiments that use the method of constant stimuli. In fact, the probit transformation apparently originated with early psychophysical investigators (Finney, 1962). When the method of constant stimuli is used in psychophysical experiments, the observer is presented with a range of stimulus intensities that are analogous to the dosage levels in a bioassay. The proportion of stimuli detected by the observer at each specific stimulus

This work was supported by NASA Grant NAG2-132 and NIH Grant 5M01 RR00088-19. B. Sakitt provided valuable comments on an earlier version of this manuscript. Reprint requests should be addressed to the author at the Department of Psychology E10-130d, Massachusetts Institute of Technology, Cambridge, Massachusetts 02139. intensity is the dependent variable. As in the bioassay, this is a quantal response variable.

In this type of a psychophysical experiment, the primary quantity of interest is usually the stimulus intensity at which the observer detects $50 \%$ of the stimuli. This threshold is analogous to the $\mathrm{LD}_{50}$ in a toxicological study. In both the bioassay and psychophysical experiments, the response functions that result have similar characteristics. If the proportion of stimuli detected or organisms responding is plotted against the dosages administered or stimulus intensities applied, a symmetrical, sigmoidal curve (a logarithmic function) usually results.

Probit analysis is a regression-like maximumlikelihood procedure for estimating the characteristics of the dosage-response curve so that the $\mathrm{LD}_{50}$ or $50 \%$ threshold and its confidence limits can be computed. The probit transformation converts the sigmoidal curve into a straight line. The slope and intercept of this line can then be estimated with a maximumlikelihood technique. A standard linear regression technique is not suitable for quantal data because the response at every dose (stimulus intensity) is a sample from a binomial population. Unlike standard linear regression techniques, probit analysis heavily weights data points near the $50 \%$ threshold point and does not assign equal weights to all the observations. A full explanation of the technique and a detailed rationale can be found in Finney (1962). A computer program for applying probit analysis to psychophysical data is presented below. It is based on Finney's (1962) methodology and, in part, on a previously published FORTRAN bioassay program (Davies, 1971).

\section{IMPLEMENTATION}

The implementation of probit analysis is written in APPLESOFT BASIC, the standard language of the Apple II microcomputer. It should be readily transferable to other computer systems. The program first requests the parameters of the analysis to be performed: 
number of stimulus levels and proportion of responses to control stimuli (Figure 1, lines 100-120). This last parameter is a correction factor for false alarms if a control condition (zero stimulus intensity) is used. If control trials are not presented, zero should be entered. The program then requests the data necessary to perform the specified probit analysis: the intensity of each stimulus level, the number of trials presented, and the number of stimuli correctly detected at that intensity. The data are then displayed, as they were input, for verification (Figure 1, lines 184-194). After all the data points have been entered and checked, the stimulus intensities are transformed logarithmically (base 10), Abbott's formula, which corrects for the number of control responses, is applied (Finney, 1962), and the empirical probits are determined. Abbott's formula is used to correct for the number of controls responding (Fig- ure 1, line 230). The probit transformation, often read from a table, is calculated by subroutine PROBIT, the method used by Davies (1971). The probit transformation, based on a cumulative normal distribution (for a detailed explanation, see Finney, 1962), transforms the sigmoidal curve into a straight line so that it can be fit to a regression line (Figure 1, lines 10001190). The next portion of the program (Figure 1, lines 300-540) accomplishes this in a manner corresponding to a graphical method of obtaining a provisional probit regression line (Davies, 1971). In cases in which stimulus intensities yield values of $0 \%$ or $100 \%$ correct, they are not used for calculation of this regression line (Figure 1, line 330).

Once a provisional regression line has been computed, it is possible to calculate the expected probits, weights, and working probits, so a more accurate regression

1 REM PROBIT ANALYSIS

10 CALL - 936: PRINT : PRINT " PROBIT ANALYSIS": PRINT

100 REM INPUT VARIABLES

105 INPUT "NAME OF EXPERIMENT? ";NAMES: PRINT

110 INPUT "NO. OF STIMULUS INTENSITIES PRESENTED ";NC: PRINT

120 INPUT "PROPORTION OF RESPONSES TO CONTROL STIMULI ";C: PRINT

140 FOR I = 1 TO NC

150 PRINT "INTENSITY OF STIMULUS\#"; I: INPUT $X(I):$ PRINT

170 INPUT "NO. STIMULI PRESENTED AT THIS INTENSITY ";AN(I): PRINT

180 INPUT "NO. OF STIMULI DETECTED AT THIS INTENSITY ";AR(I): PRINT

182 NEXT I

184 PRINT" DATA AS INPUT"

186 PRINT : PRINT "INTENSITY";" NO. PRESENT NO. OETECT"

188 FOR $M=1$ TO NC

190 PRINT X(M), AN (M), AR (M)

192 NEXY M

194 INPUT "HIT RETURN TO CONTINUE"; XX\$

200 REM TRANSFORM CONCENTRATIONS, COMPUTE EMP. PROBITS BY ABBO TTS FORMULA

210 FOR $I=1$ TO NC

$220 \times(\mathrm{I})=$ LOG $(X(1))$

$222 X(\mathrm{I})=X(\mathrm{I}) * 0.434394:$ REM CONVERT NATURAL TO COMMON LOG

$230 \mathrm{P}(\mathrm{I})=($ AR $(\mathrm{I}) / \mathrm{AN}(\mathrm{I})-\mathrm{C}) /(1.0-\mathrm{C})$

240 GOSUB 1000: REM GET PROBIT VALUE

$242 Y(I)=$ PROBIT

250 NEXT I

300 REM COMPUT PROV REGRESS LINE

$303 X Y S=0: S X=0: S Y=0: X S X=0$

$310 \mathrm{DEN}=\mathrm{NC}$

320 FOR I $=1$ TO NC

330 IF $Y(I)>-1.0 E 25$ ANO $Y(I)<1.0$ E 25 THEN 400

340 DEN $=$ DEN -1.0

350 60TO 440

$400 X Y S=X Y S+X(I) * Y(I)$

$410 S X=S X+X(I)$

$420 \mathrm{SY}=S Y+Y(I)$

$430 \times S 2=X S 2+X(I) * X(I)$

440 NEXT

$500 X S 2=X S 2-S X * S X / D E N$

$510 X Y S=X Y S-S X * S Y /$ DEN

$520 \mathrm{~B}=X Y S / X S 2$

$530 \mathrm{~A}=S Y / D E N-B * S X / D E N$

540 YCNC $=0$

600 REM COMPUTE EXPECTED PROBITS, WEIGHTS, AND WORKING PROBITS

602 FOR $I=1$ TO NC

$604 Y(I)=B * X(I)+A$

$610 Z=0.3989423 * \operatorname{EXP}\left(-0.5 *(Y(I)-5.0)^{\wedge} 2\right)$

$620 P 1=Y(I)-5.0$

630 GOSUB 2000

640 P1 = PN: REM PN FROM SUBROUTINE

649 REM WN IS WEIGHTING COEF

650 WN $(1)=A N(I) * Z * Z /(1.0-P 1) /(P 1+C /(1.0-C))$

$680 Y(I)=Y(I)+(P(I)-P 1) / 2$

690 NEXT

700 REM COMPUTE WEIGHTED REGRESSION

$702 \$ 1=0: \$ 2=0: \$ 3=0: \$ 4=0: 55=0: S 6=0$

706 FOR $1=1$ TO NC

$710 \mathrm{S1}=\mathrm{S} 1+\mathrm{WN}(\mathrm{I})$

$720 \mathrm{S2}=\mathrm{S} 2+\mathrm{WN}(\mathrm{I}) * X(\mathrm{I})$

$73053=S 3+W N(1) * Y(I)$

$74054=54+W N(1) * X(I) * Y(1)$

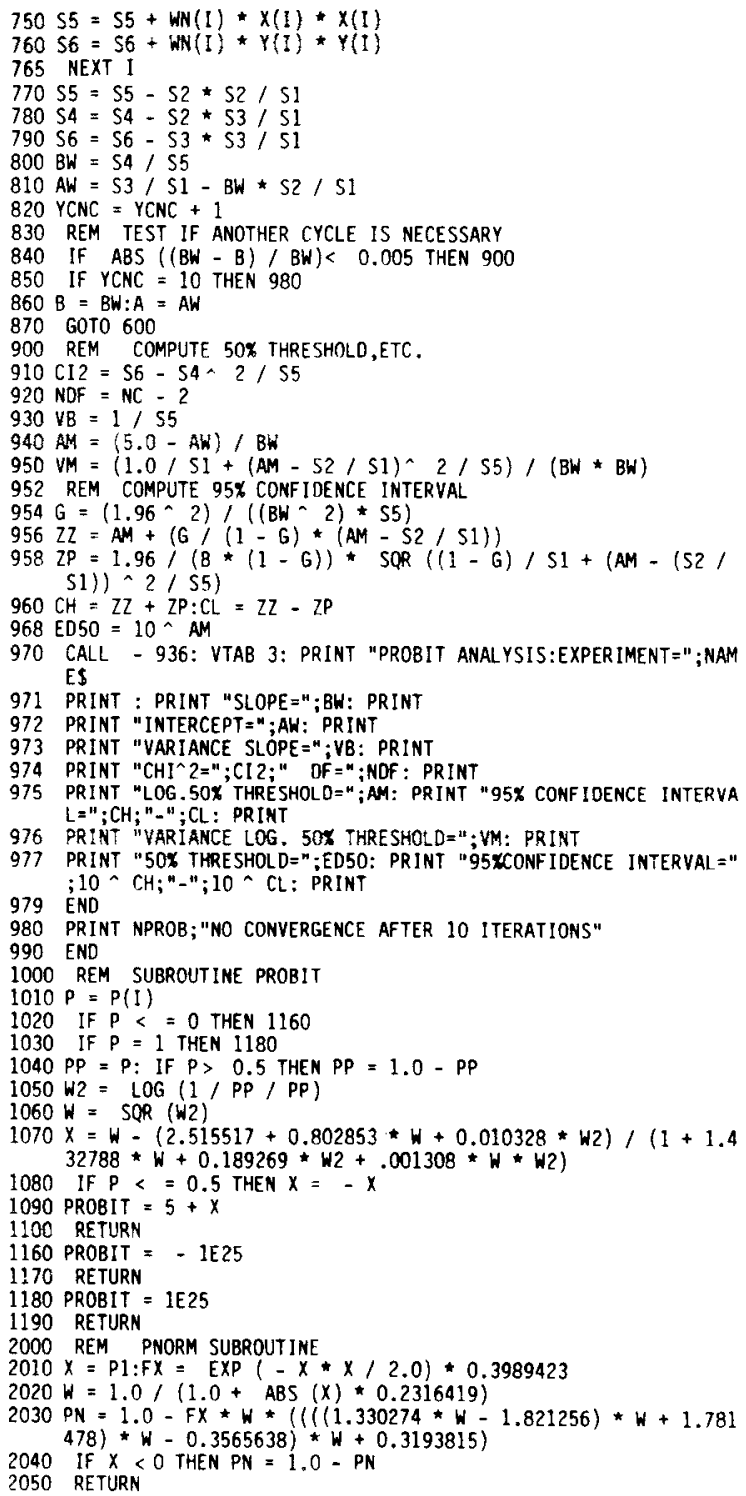

Figure 1. The program for the computation of probit analyses on psychophysical data that is described in the text. 
DATA AS INPUT

\begin{tabular}{|c|c|}
\hline $\begin{array}{lc}\text { INTENSITY } & \text { NO. PRESENT } \\
2.6 & 50 \\
3.8 & 48 \\
5.1 & 46 \\
7.7 & 49 \\
10.2 & 50 \\
\text { HIT RETURN TO CONTINUE } \\
\text { PROBIT ANALYSIS: EXPERIMEN }\end{array}$ & $\begin{array}{l}\text { NO. DETECT } \\
6 \\
16 \\
24 \\
42 \\
44 \\
\text { FINNEY EX.6 }\end{array}$ \\
\hline \multicolumn{2}{|l|}{$S L O P E=4.21222898$} \\
\hline \multicolumn{2}{|l|}{ INTERCEPT $=2.11253811$} \\
\hline \multicolumn{2}{|l|}{ VARIANCE SLOPE $=.23084736$} \\
\hline \multicolumn{2}{|l|}{$\mathrm{CHI}^{\wedge} 2=1.72902615$ DF $=3$} \\
\hline \multicolumn{2}{|c|}{$\begin{array}{l}\text { LOG. 50X THRESHOL }=.685494996 \\
95 \times \text { CONF IDENCE INTERVAL }=.728875706-.640081524\end{array}$} \\
\hline \multicolumn{2}{|c|}{ VARIANCE LOG. 50X THRESHOLO $=4.87251323 E-04$} \\
\hline $\begin{array}{l}\text { 50\% THRESHOLD }=4.84724528 \\
\text { 95\%CONF IDENCE INTERVAL }=5\end{array}$ & $43336-4.36597781$ \\
\hline
\end{tabular}

Figure 2. Input and output data from the probit computer program presented in Figure 1. The data used were taken from Finney (1962). The "proportion of responses to control stimuli" equals zero in this example.

line can be computed (Figure 1, line 600-690). The iterative procedure necessary for these computations requires subprogram "PNORM" (Figure 1, lines 20002050), which calculates the area beneath the normal curve (Davies, 1971). The improved regression line is then determined by a maximum-likelihood method (Figure 1, line 700-820). The computations are terminated when the relative error of the regression line is less than 0.005 (Figure 1, line 840). If after 10 iterations have been performed the relative error is greater than 0.005 , the program is terminated without computing the final results (Figure 1, line 850).
Finally, in lines 900 to 979 (Figure 1), the results are computed and printed out. Of particular interest is the threshold, but, in addition, the log threshold, its variance, the slope and intercept of the regression line, the variance of the slope, and the chi-square statistic are provided. Confidence intervals (95\%) for both the threshold and the logarithmic threshold are also computed.

A sample data set from Finney (1962, Example 6) and the output generated by the program when this data set is entered are provided in Figure 2.

\section{REFERENCES}

Buiss, C. I. The calculation of the dosage mortality curve. Annals of Applied Biology, 1935, 22, 134-167.

Bock, R. D., \& Jones, L. V. The measurement and prediction of judgment and choice. San Francisco: Holden-Day, 1968.

Davies, R. G. Computer programming in quantitative biology. London: Academic Press, 1971.

Finney, D. J. Probit analysis (2nd. ed.). Cambridge: Cambridge University Press, 1962.

Garwood, F. The application of maximum likelihood to dosagemortality curves. Biometrika, 1941, 32, 46-58.

Sakitr, B. Canonical ratings. Perception \& Psychophysics, 1974, 16, 478-488.

Yang, J. C., Clahk, W. C., Ngai, S. H., Berkowitz, B. A., \& SPECTOR, S. Analgesic action and pharmacokinetics of morphine and diazepam in man: An evaluation by sensory decision theory. Anesthesiology, 1979, 51, 495-501.

\section{NOTE}

1. See the Appendix by R. A. Fisher, in Bliss (1935).

(Manuscript received March 4, 1983; revision accepted for publication June 2,1983 .) 